\title{
Reconstrucción de 350 años de precipitación para el suroeste de Chihuahua, México
}

\author{
Reconstructing 350 years of precipitation in southeast \\ Chihuahua, Mexico
}

\author{
Julián Cerano Paredes1, José Villanueva Díaz1, \\ Peter Z. Fulé2, Jesús Guadalupe Arreola Ávila3, \\ Ignacio Sánchez Cohen ${ }^{1}$ y Ricardo David Valdez Cepeda ${ }^{3}$
}

\author{
RESUMEN
}

\begin{abstract}
En el suroeste del estado de Chihuahua, México, en la reserva "Cerro El Mohinora" se desarrollaron series de tiempo dendrocronológicas de madera temprana, tardía y anillo total de Pseudotsuga menziesii con una longitud de tres siglos y medio (1657-2005), con el objetivo de analizar la variabilidad hidroclimática histórica de la región. La reconstrucción de precipitación invierno-primavera indica gran variabilidad interanual, decenal y multidecenal de los patrones de precipitación para la región. Sequías severas se reconstruyeron para los periodos 1695-1715, 1753-1760, 1785-1792, 1798-1806, 1819-1830, 1841-1870, 1890-1897, 1906-1912, 1924-1941, 1971-1977 y 1994-2005, aunque las sequías más prolongadas ocurrieron en los periodos 1695-1715, 1841-1870 y 1924-1941. Las últimas tres décadas del siglo XX y los años corrientes de la primera década del siglo XXI (1971-2005) indican un periodo de intensa sequía para la región, con impactos en lo ecológico y socioeconómico aun no cuantificados.
\end{abstract}

PALABRAS CLAVE:

Madera tardía, madera temprana, Pseudotsuga menziesii, reconstrucción de precipitación.

\begin{abstract}
Earlywood, latewood, and total ring width series from Pseudotsuga menziesii were developed in a mixed conifer forest at Cerro El Mohinora", Chihuahua. The tree-ring series extended for the period 1657-2005 (349 years) and were used to analyze hydroclimatic variability for southwestern Chihuahua. A seasonal winter-spring precipitation reconstruction was developed with the objective to determine high and low frequency precipitation variability and to analyze the impact of dominant atmospheric circulatory patterns. The reconstruction indicated high interannual, decadal and multiannual variability. Severe droughts were reconstructed for the periods 1695-1715, 1753-1760, 1785-1792, 1798-1806, 1819-1830, 1841-1870, 1890-1897, 1906-1912, 1924-1941, 1971-1977, and 1994-2005. The most extended droughts took place in the periods 1695-1715, 1841-1870, and 1924-1941. The last three decades of the $20^{\text {th }}$ century and the current years of the $21^{\text {st }}$ century have been particularly dry for northwestern Chihuahua but the ecological and socieoconomical impacts have not been determined.
\end{abstract}

KEY WORDS:

Latewood, earlywood, Pseudotsuga menziesii, precipitation reconstruction.

Instituto Nacional de Investigaciones Forestales, Agrícolas y Pecuarias. Centro Nacional de Investigación Disciplinaria Relación Agua-Suelo-Planta-Atmósfera. km 6.5 margen derecha Canal Sacramento Gómez Palacio, Dgo. Apdo. Postal 41. Código Postal 35150. Lerdo, Dgo. Email: cerano.julian@inifap.gob.mx

2 Northern Arizona University, School of Forestry and Ecological Restoration Institute, P. O. bOX 15018, Flagstaff, AZ 86011,USA. pete.fule@nau.edu

3 Unidad Regional Universitaria de Zonas Áridas, Universidad Autónoma Chapingo. Domicilio conocido Bermejillo, Durango. Código Postal 35230. Email: vacrida@gmail.com 


\section{INTRODUCCIÓN}

Hoy en día, el entendimiento de la variabilidad del clima es uno de los temas más relevantes de las ciencias ambientales. El clima varía de un año a otro, entre décadas e incluso entre milenios. La actividad industrial y los gases de efecto invernadero liberados a la atmósfera a consecuencia de la actividad antropogénica, han alterado el clima de la tierra (Houghton et al., 1990). En este sentido, se han producido cambios en los patrones de precipitación asociados al calentamiento global (Bradley et al., 1987, Díaz et al., 1989), alterando el comportamiento natural del ciclo hidrológico (IPCC, 1996). Aunque no existen evidencias claras a escalas locales sobre los efectos del calentamiento global de la tierra, existe consenso científico en cuanto a que hay una gran incertidumbre con respecto a la magnitud y tasas de estos cambios a escalas regionales (EEI, 1997).

Los registros del clima procedentes de satélites y registros instrumentales cubren por lo general menos de 80 años. Estos registros son demasiado cortos para examinar el rango completo de la variabilidad climática. Existen diferentes fuentes naturales indirectas que registran dicha variación en el tiempo, tales como: anillos de árboles, capas de hielo, polen fósil, sedimentos marinos, corales y documentos históricos. Al analizar los registros tomados de éstas y de otras fuentes paleoclimáticas, se puede extender el conocimiento de la variación del clima mucho más allá de los registros climáticos instrumentales (Bradley, 1999).

Los anillos de árboles constituyen la fuente indirecta más apropiada de resolución anual para entender el comportamiento histórico del clima, su crecimiento anual está influenciado por las condiciones climáticas dominantes previas o durante su formación. Son una herra- mienta para el análisis de las condiciones climáticas en periodos en los que no existen registros instrumentales (Fritts, 1976).

Los bosques templados de la Sierra Madre Occidental poseen coníferas con excelente potencial dendrocronológico, en el que el desarrollo tanto de madera temprana como tardía está influenciado por las condiciones dominantes de precipitación de invierno y de verano, que a su vez son modulados por patrones atmosféricos de circulación global (Stahle et al., 1998).

Los estudios de variabilidad hidroclimática han sido poco desarrollados en la región de Chihuahua (Díaz et al., 2002; Villanueva-Díaz et al., 2006). La reserva Cerro El Mohinora, al suroeste del estado de Chihuahua, presenta ecosistemas de bosques mixtos con especies de excelente potencial dendroclimático como es el caso de Pseudotsuga menziesii, representando estos sitios un gran potencial para reconstruir la precipitación, información que ayudará a un mejor entendimiento de la variabilidad hidroclimática a nivel regional y a entender su impacto en los ecosistemas.

\section{OBJETIVOS}

1) Generar una serie dendrocronológica con base en los anillos de crecimiento de Pseudotsuga menziesii.

2) Reconstruir la precipitación de los últimos tres siglos y medio y analizar su variabilidad hidroclimática en el tiempo.

\section{METODOLOGÍA}

\section{Localización del área}

El sitio Cerro El Mohinora se localiza al suroeste del estado de Chihuahua, 
cerca de los límites con los estados de Durango y Sinaloa (Figura 1). El área comprende un gradiente de elevación de 2500 a $3000 \mathrm{~m}$, donde se desarrolla un bosque de pino y Abies-PseudotsugaPicea en la parte alta (250 57' 16" N, 1070 01' 38" W). En la parte media se localiza un bosque mixto de Abies-Pseudotsuga-Picea (250 58' 27' N, 1070 02' 26 " W) y en la parte baja bosque de pino (250 58' 23" N, $107001^{\prime} 35^{\prime \prime} \mathrm{W}$ ).

El área presenta temperaturas promedio máximas de 25 a $270 \mathrm{C}$ para los meses de mayo a septiembre; temperaturas promedio mínimas de -1 a $-3^{0} \mathrm{C}$ para los meses de diciembre a marzo. Registra una precipitación promedio para los meses más lluviosos del año de $110 \mathrm{~mm}$ en junio, $274 \mathrm{~mm}$ en julio, $249 \mathrm{~mm}$ en agosto, $164 \mathrm{~mm}$ para septiembre y un promedio anual de $1130 \mathrm{~mm}$. Los promedios de las variables se generaron de un periodo de 25 años (ERIC II, 2000).

Para reconstruir la variabilidad climática de la región suroeste del estado de Chihuahua, se tomaron núcleos de crecimiento y secciones transversales de Pseudotsuga menziesii. Se empleó un muestreo selectivo; en cada árbol seleccionado, a la altura del pecho y perpendicular a la pendiente, con taladro Pressler de $5 \mathrm{~mm}$ de diámetro interno y extensiones variables, se extrajeron dos virutas (núcleos de crecimiento) por árbol (Figura 2); asimismo, con motosierra se cortaron secciones transversales de tocones, árboles muertos o madera subfósil (secciones de madera sobre la superficie

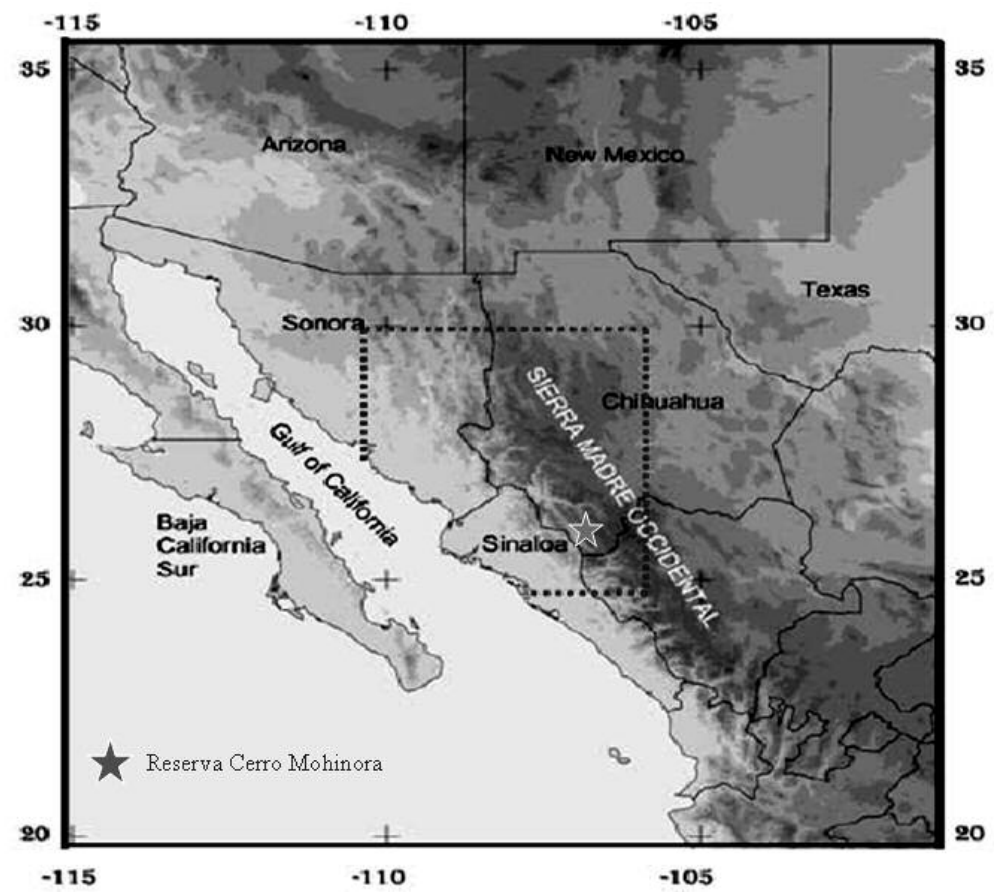

Figura 1. Localización geográfica del sitio de muestreo en la reserva "Cerro El Mohinora" al suroeste del estado de Chihuahua. 


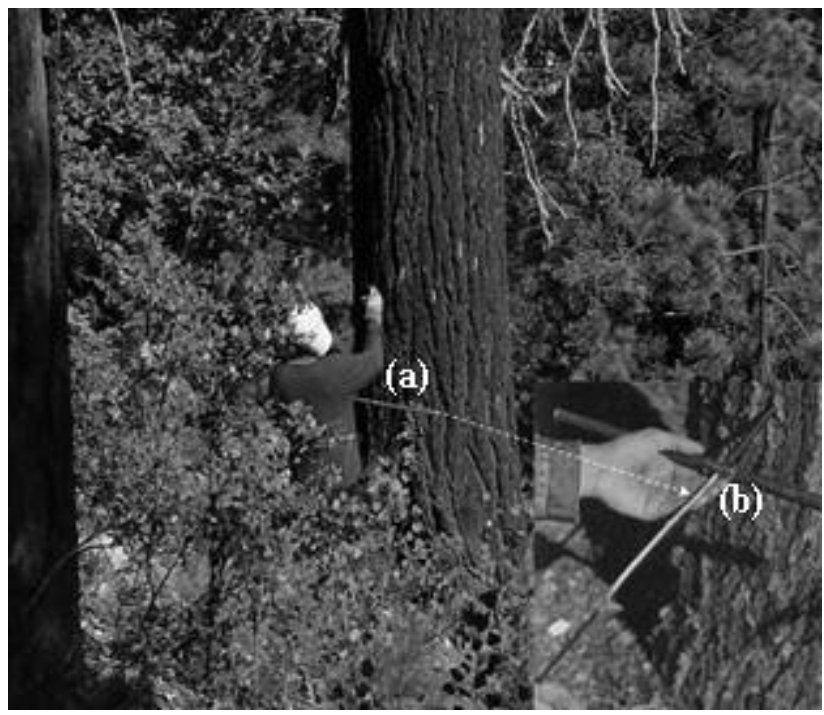

Figura 2. Toma de muestras de árboles vivos (virutas o núcleos de crecimiento) empleando el Taladro de Pressler, a) muestra la posición de la barrena para la toma de la muestra y b) la forma en que se extrae el núcleo de crecimiento.

del suelo o semienterrada). Los núcleos de crecimiento se identificaron, montaron y pegaron en secciones acanaladas de madera para facilitar su manejo.

Las muestras se pulieron con diversos grados de lija (del número 30 la más gruesa, a la más fina del número 1200) para resaltar sus estructuras de crecimiento. En el Laboratorio de Dendrocronología del INIFAP CENID-RASPA se contaron los anillos con el apoyo de un estereomicroscopio de alta resolución, los anillos se contaron y fecharon al año exacto de su formación con base en técnicas dendrocronológicas estándar (Stokes y Smiley, 1968).

El principio del fechado cruzado es el principio básico de la dendrocronología. Es la herramienta principal por medio de la cual es determinado el crecimiento exacto de cada uno de los anillos anuales. Un simple conteo de anillos posiblemente generaría errores debido a que existen anillos ausentes (perdidos) y falsos anillos (dobles). El fechado cruzado es imprescindible cuando las mediciones del ancho de los anillos son comparadas con alguna variable climática anual o estacional, tal como precipitación, temperatura, presión atmosférica, etc. Sin un fechado preciso de los anillos de crecimiento, es imposible una calibración adecuada debido a que la cronología estará desfasada en el tiempo. Esta técnica permite observar en una muestra, donde existen anillos perdidos o donde podríamos tener formados dos o más anillos en un año. El fechado al año de formación de cada uno de los crecimientos anuales es el objetivo principal de esta técnica (Figura 3).

Una vez fechadas las muestras, se midieron individualmente las bandas de madera temprana, tardía y anillo total con un sistema de medición VELMEX, equipo 


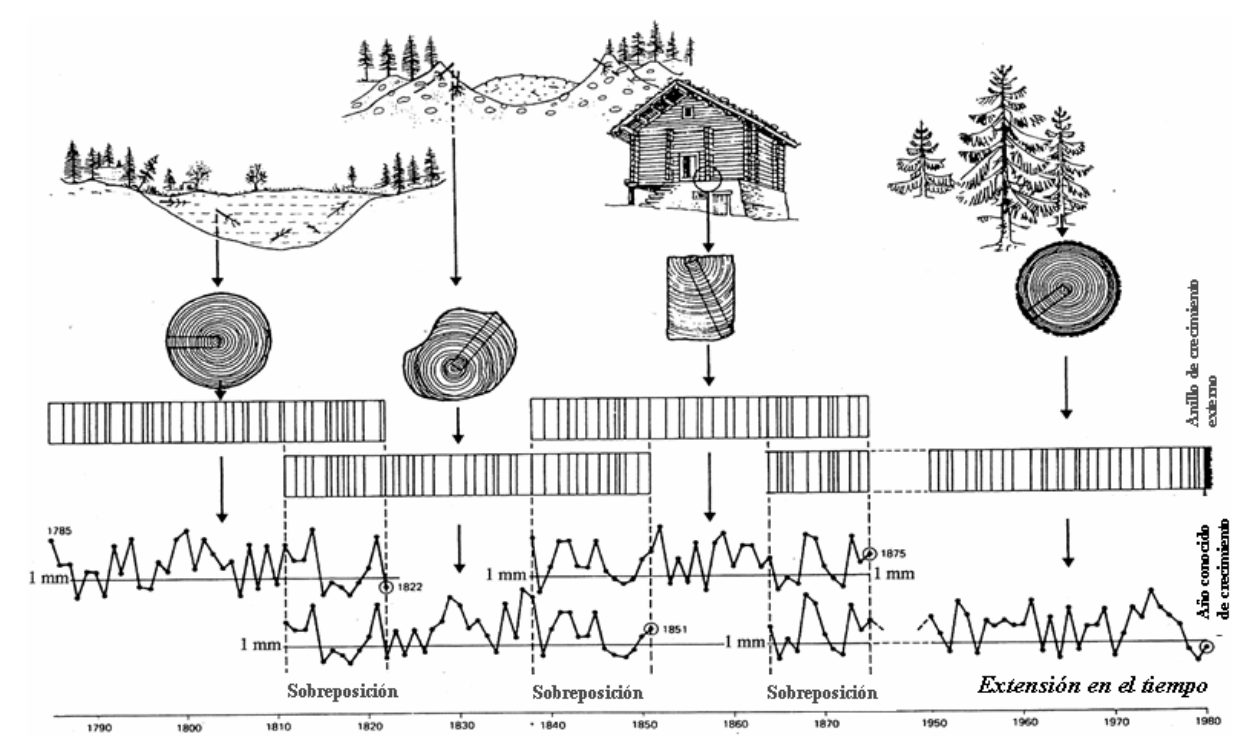

Figura 3. Fechado Cruzado, Principio de la Dendrocronología, que permite determinar el año exacto de formación de cada anillo de crecimiento y que con base en la similitud de los patrones de crecimiento es factible extender en el tiempo las series

dendrocronológicas, al lograr datar árboles vivos, troncos muertos, enterrados o semienterrados, que a su vez, determinarán la extensión en el tiempo para propósitos de reconstrucción climática (Fritts, 1976).

compuesto por una platina deslizable y un codificador linear que tiene una precisión de medición de $0.001 \mathrm{~mm}$, sistema integrado a un procesador PC y un estereomicroscopio con una retícula montada en el ocular para ubicar los límites de cada anillo de crecimiento.

Después de generada la base de datos, se verificó la calidad del fechado, la respuesta climática y la exactitud de la medición de cada anillo con el programa COFECHA (Holmes, 1983). De las series de crecimiento, las tendencias biológicas como competencia y liberación y las geométricas (el área de fuste se incrementa con la edad y el crecimiento anual tiende a disminuir al distribuirse en una mayor superficie) no relacionadas con clima, se removieron con el programa
ARSTAN, al insertar una curva exponencial negativa o línea recta a la serie de medición y luego al dividir cada valor anual de medición entre el valor obtenido de la curva. Esto creó una serie de índices normalizados (cronologías) con media de 1,0 y varianza homogénea (Cook, 1987).

Para conocer el periodo de lluvia anual que influye de manera significativa en el crecimiento del arbolado, se utilizaron datos de la estación climática Guadalupe y Calvo, Chihuahua, (Instituto Mexicano de Tecnología del Agua, 2000) que es la estación más cercana al área de estudio. La respuesta climática o Análisis de Función de Respuesta se investigó al relacionar los índices de anillo con los datos observados de precipitación empleando el programa PRECON (Fritts, 1999). 
Definido el periodo de respuesta climática, con los datos climáticos disponibles y los índices de anillo de madera temprana para el mismo periodo, se generó un modelo de predicción. El modelo se aplicó para generar la precipitación de este mismo periodo. Para lo anterior en la mitad de los datos se realizó una calibración entre la precipitación observada y la generada, y en la otra mitad se realizó una verificación (Fritts, 1991). Finalmente, se aplicó la ecuación de transferencia del periodo total de datos de precipitación disponible para desarrollar la reconstrucción de precipitación en la longitud total de la cronología. A la serie de alta frecuencia (resolución anual) se le ajustó una curva decenal flexible (baja frecuencia) para resaltar eventos secos o húmedos (Cook y Peters, 1981).

La reconstrucción de precipitación generada se comparó con otras reconstrucciones realizadas para el norte de México. Los periodos de sequía presentes en la reconstrucción se compararon con periodos similares en otras reconstrucciones de precipitación realizadas para sitios ubicados en las sierras Madre Oriental y Occidental. En la medida de lo posible, se analizaron archivos históricos que nos permitieron verificar la calidad de la reconstrucción realizada; asimismo, se analizó la influencia de fenómenos atmosféricos circulatorios como El Niño, Oscilación del Sur (ENSO, por sus siglas en inglés) y el Monzón de Norte América (MNA) en la variabilidad climática de la región.

\section{RESULTADOS}

\section{Reconstrucción de la variabilidad climá- tica para el suroeste de Chihuahua}

Con el análisis de 53 núcleos de crecimiento se desarrollaron cronologías de madera temprana, tardía y anillo total con una extensión de 349 años, que corresponden al periodo de 1657 a 2005. Según el programa COFECHA la cronología de madera temprana tuvo una intercorrelación entre series alta ( $r=0,61, p<0.05)$, lo cual es indicativo de una respuesta climática común (Holmes, 1983).

\section{Función de respuesta}

Para definir la respuesta climática de la cronología se utilizó el programa PRECON (Fritts, 1999). El programa usado produjo un análisis de función de respuesta con base en los índices de crecimiento y los registros de precipitación disponibles. En este caso, para el periodo 1966-1985, se determinó a qué periodo de precipitación está respondiendo el crecimiento de los árboles del sitio. La función de respuesta incluye 14 meses de precipitación de julio del año previo a agosto del año en curso, que comprende la estación de crecimiento. Tres elementos adicionales están asociados con el índice de anillo, el crecimiento de 1, 2 y 3 años previos.

Esto permitirá determinar si el crecimiento actual está influido por el crecimiento de años previos y no sólo por la variable climática. Un coeficiente significativo indica una relación directa de la variable clima o crecimiento previo con el ancho del anillo y un coeficiente negativo indica una relación inversa. Las líneas verticales indican un nivel de significancia del $95 \%(p<0,05)$ (Figura 4$)$.

Los meses individuales de precipitación acumulada que se correlacionaron significativamente $(p<0,05)$ con la cronología de madera temprana son: diciembre del año anterior, marzo, abril, mayo, julio y agosto del año actual de crecimiento (Figura 4). 


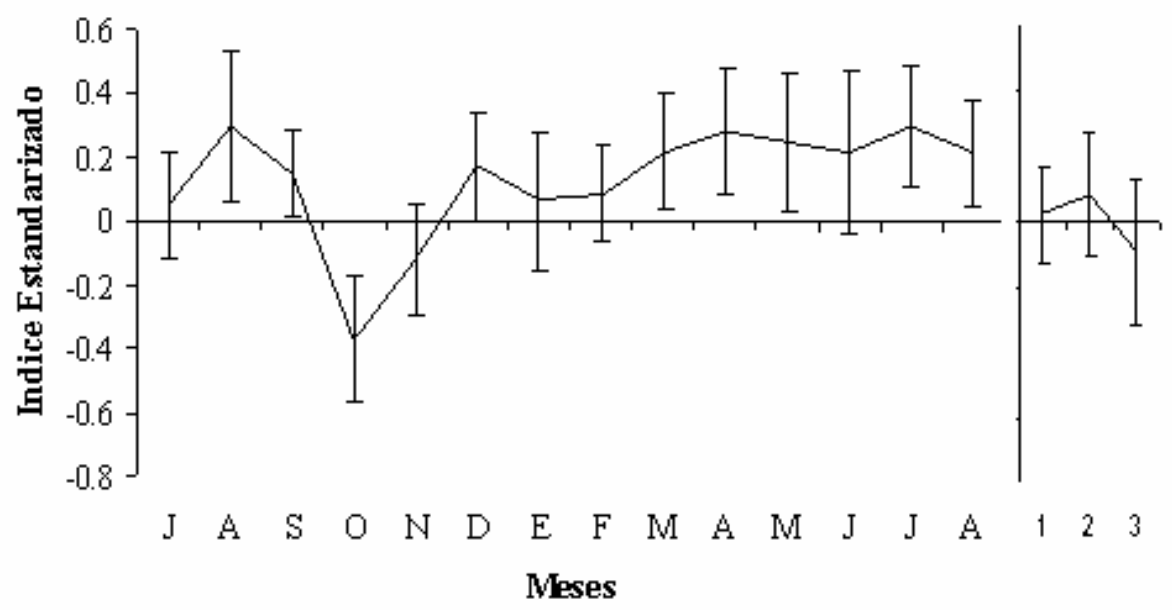

Figura 4. Función de respuesta entre el índices de madera temprana de Pseudotsuga menziesii y los registros de precipitación para el período 1966-1985 de la estación climática Guadalupe y Calvo, Chihuahua.

No obstante que la correlación entre el índice de madera temprana y precipitación para los meses de enero, febrero y junio presentan una relación positiva pero no significativa $(p>0,05)$, se encontró una respuesta significativa $(p<0,05)$ al periodo estacional enero-junio. Para los tres años previos asociados al análisis de respuesta se encontró que las condiciones climáticas previas no influyen de manera significativa $(p>0,05)$ en el crecimiento anual.

\section{Reconstrucción de la precipitación}

Al relacionar la cronología de madera temprana con datos instrumentales del periodo de precipitación estacional enero-junio, periodo 1966-1985, se encontró una respuesta significativa entre ambas variables con una correlación de $0.74(p<0.001)$, lo que sugiere un potencial adecuado para la reconstrucción de la precipitación (figuras 5 y 6 ).

\section{Calibración y verificación}

Para que un modelo de reconstrucción sea estadísticamente válido, es preciso desarrollar dos procesos; uno de calibración, realizado en la mitad de los datos climáticos disponibles y uno de verificación, en la mitad restante. La calibración es la asociación entre dos variables. En este caso el índice de la cronología de ancho de anillo es una variable y los datos promedio mensuales de precipitación representan los valores de la variable climática con los cuales el índice se calibrará. El éxito de la calibración se mide por el porcentaje de la variación que la variable independiente (índice de madera temprana) explica de la variable dependiente reconstruida (precipitación). Por otra parte, la verificación tiene como objetivo validar la reconstrucción, mientras que la calibración únicamente examina la relación entre ambas variables. 
.... In dice de Madera Temprana — Precipitación

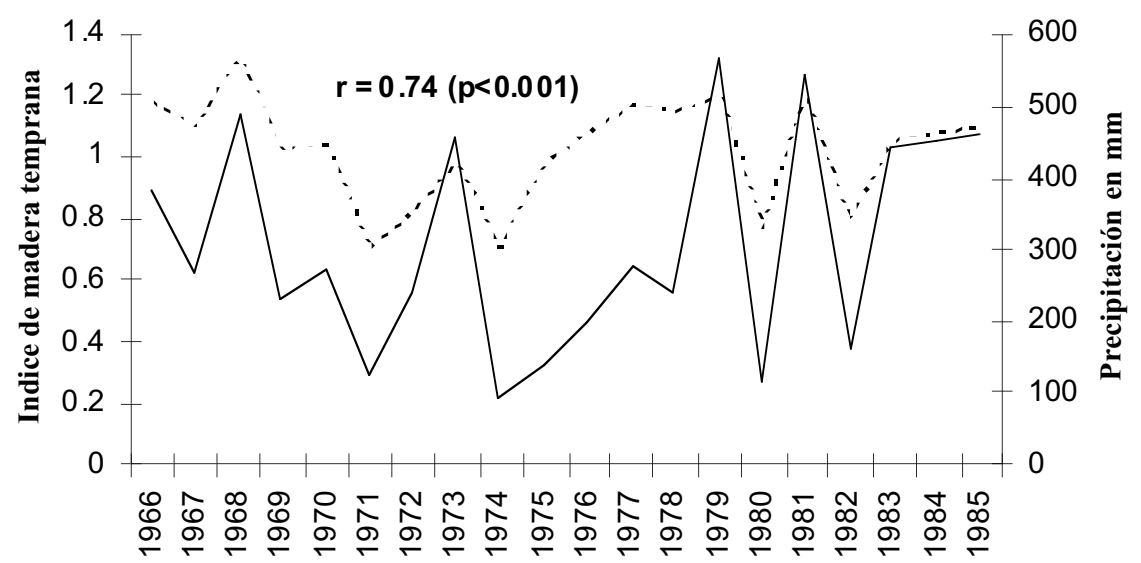

Año

Figura 5. Asociación entre el índice de madera temprana y precipitación estacional observada para el período enero-junio, que comprende el intervalo de 1966-1985.

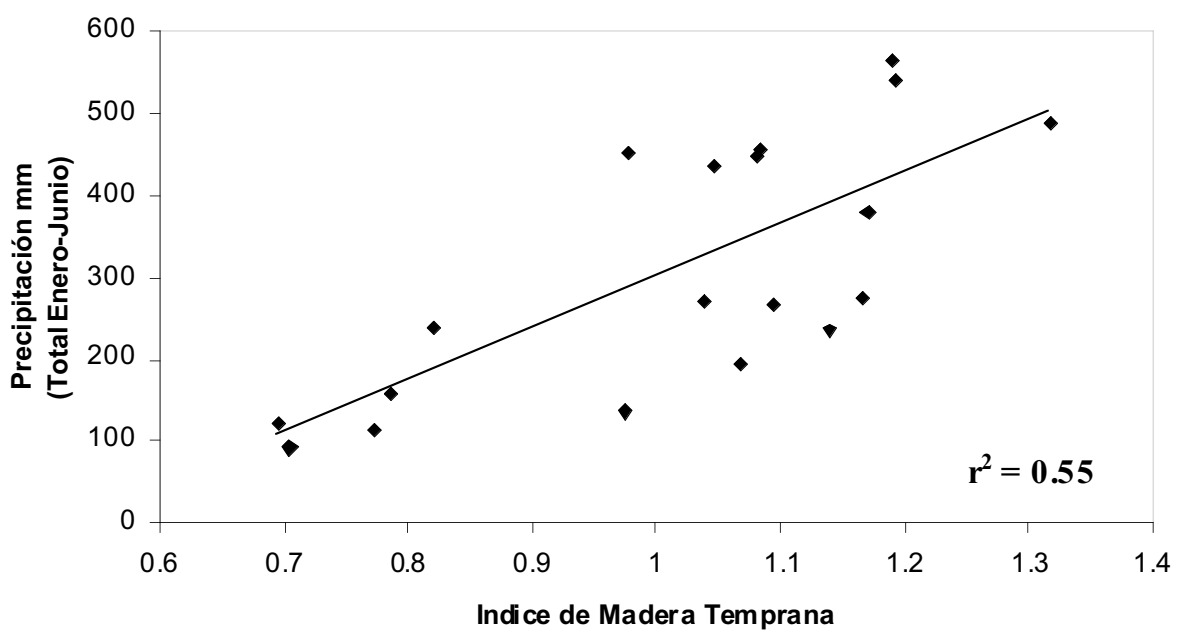

Figura 6. Gráfico de dispersión del modelo de regresión lineal que muestra la variabilidad de la cronología de madera temprana con la precipitación total de enerojunio, período 1966-1985. 
El modelo utilizado para la reconstrucción incluyó el periodo total de datos disponibles (1966-1985) y los índices de madera temprana para el mismo periodo. Una vez aplicado el modelo, éste se calibró con datos del subperiodo 19741985 y se verificó en el subperiodo 19661973.

Los resultados del modelo indican una correlación de $r=0,72\left(r^{2}=0,52, p<0,001\right)$ entre la precipitación observada y la precipitación reconstruida, y explica $52 \%$ de la variabilidad en precipitación para el periodo de calibración. La verificación presentó una correlación de $r=0,88$ y explica $77 \%\left(r^{2}=\right.$ $0,77, p<0,001)$ de la variabilidad. El periodo total de precipitación observada y reconstruida muestra una $r=0,76\left(R^{2}=0 ., 58\right.$, $\mathrm{p}<0,001$ ) (Figura 7).

Debido a que los subperiodos utilizados tanto para realizar la calibración como la verificación del modelo entre la precipitación observada y reconstruida estuvieron correlacionados significativamente, se utilizó todo el periodo de datos climáticos disponibles (1966-1985) para generar la ecuación de reconstrucción que mostró una $r=0,74 \quad\left(r^{2}=0,55\right.$, $\mathrm{p}<0,001)$ (tablas 1 y 2 ).

La subrutina VERIFY5 de la Base de Programas Dendrocronológicos de la Universidad de Arizona (DPL, por sus siglas en inglés), indicó que tanto la calibración como la verificación pasaron la prueba de significancia $(p<0,05)$ para la correlación, reducción de error, valor de "t" y primera diferencia significativa. El modelo de regresión obtenido para el periodo 1966-1985 se consideró estadísticamente válido para reconstruir el periodo total de la serie dendrocronológica.

El modelo lineal bivariado utilizado fue el siguiente:

$$
Y t=-374,647+670,357 * X t
$$

Donde: $Y t=$ Valor de precipitación reconstruido enero-junio para un año específico $(\mathrm{mm}) . \mathrm{Xt}=$ Índice de madera temprana.

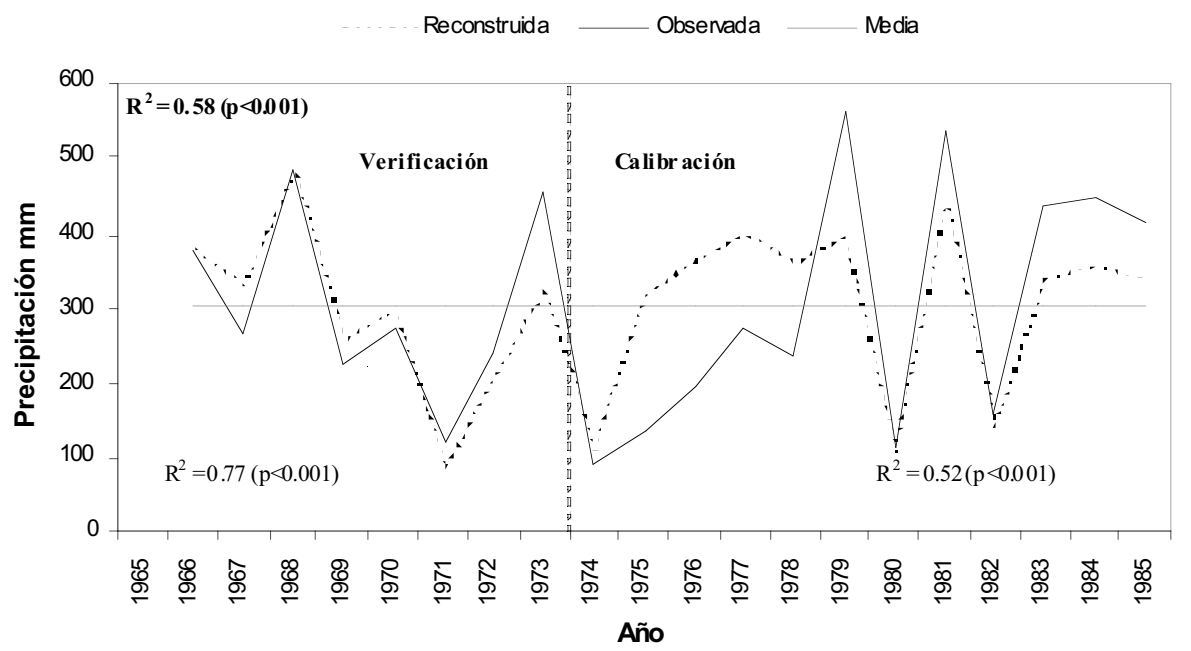

Figura 7. Precipitación observada (línea sólida) y reconstruida (línea punteada) para el período estacional enero-junio, mostrando una $r=0.76\left(R^{2}=0.58, p<0.001\right)$ para el período total (1966-1985). 
Tabla 1. Modelo lineal generado para la reconstrucción estacional de precipitación invierno-primavera.

\begin{tabular}{cccccc}
\hline Periodo & $R^{2}$ & Coeficientes & $\begin{array}{c}\text { Error } \\
\text { estándar }\end{array}$ & $\begin{array}{c}\text { Prueba de } \\
\text { " } t \text { “ }\end{array}$ & Probabilidad \\
\hline \multirow{2}{*}{$1966-1985$} & \multirow{2}{*}{0.55} & a -374.647 & 137.74 & -2.71 & 0.014 \\
& & b 670.357 & 134.44 & 4.98 & 0.000 \\
\hline
\end{tabular}

Tabla 2. Análisis de varianza del modelo de reconstrucción

\begin{tabular}{cccccc}
\hline Periodo & $\begin{array}{c}\text { Suma de } \\
\text { cuadrados }\end{array}$ & $d f$ & $\begin{array}{c}\text { Cuadrados } \\
\text { medios }\end{array}$ & $f$ & $p$ \\
\hline Regresión & 247357.4 & 1 & 247357.4 & 24.86059 & .000096 \\
Residual & 179096.1 & 18 & 9949.8 & \\
Total & 426453.5 & & & \\
\hline
\end{tabular}

\section{Análisis de la variabilidad climática}

La reconstrucción de precipitación invierno-primavera para la reserva Cerro El Mohinora, indica gran variabilidad interanual (alta frecuencia), decenal y multidecenal (baja frecuencia) de los patrones de precipitación para la región (Figura 8).

Sequías severas se reconstruyeron para los periodos $1695-1715,1753-1760$, 1785-1792, 1798-1806, 1819-1830, 18411870, 1890-1897, 1906-1912, 1924-1941, 1971-1977 y 1994- 2005. Las sequías más prolongadas ocurrieron en los periodos 1695-1715, 1841-1870, 19241941. Las últimas tres décadas del siglo XX y los años actuales de la primera década del siglo XXI (1971-2005) indican un periodo de intensa sequía para la región, que continúa hasta el presente, afectando el suroeste de Chihuahua. De igual manera, se reconstruyeron eventos con precipitaciones por arriba de la media regional, sobresaliendo los periodos de
1673-1695, 1735-1753, 1759-1785, 18091818, 1829-1839, 1898-1906, 1911-1922, 1941-1969 y finalmente de la década de los setentas, a la fecha sobresalen dos periodos con precipitaciones por encima de la media 1976-1981 y 1990-1993 (Figura 8).

\section{DISCUSIÓN}

\section{Respuesta climática}

La reconstrucción de precipitación se fundamentó en la madera temprana como variable independiente, cuya formación está ligada significativamente $(p<0.05)$ con la precipitación invernal y ésta a su vez por la fase cálida de ENSO (Ropelewski y Harper, 1989; Stahle et al., 1998 y Magaña et al., 1999). En México esta relación se registra de manera clara en la porción de madera temprana de Pseudotsuga menziesii en rodales de la Sierra Madre Occidental (Stahle et al., 1998 y Cleaveland et al., 2003). 


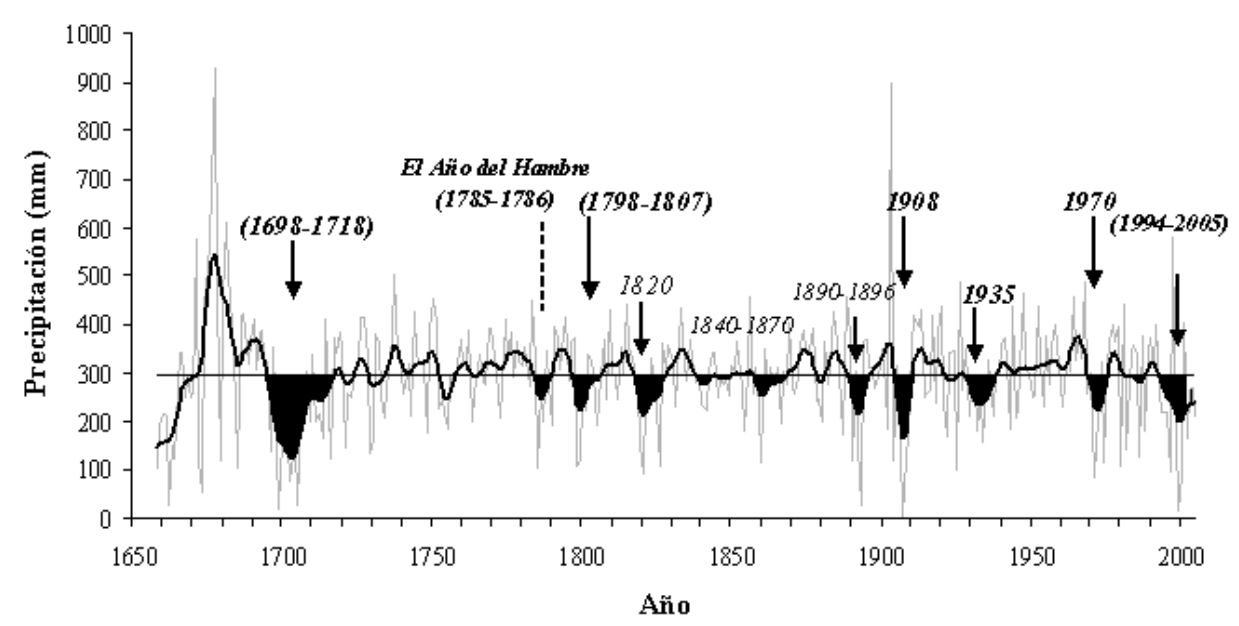

Figura 8. Precipitación estacional reconstruida para los últimos 350 años en la reserva ecológica Cerro El Mohinora, Chihuahua. La línea de color gris en el fondo indica la variabilidad anual de la precipitación, la curva flexible de color negro es una línea suavizada de 10 años para observar de mejor manera los eventos secos (precipitación por debajo de la media) y húmedos (precipitación por arriba de la media); la línea recta muestra la precipitación promedio $(294 \mathrm{~mm})$. Las sequías reconstruidas más intensas que fueron verificadas con archivos históricos y otros trabajos de reconstrucción de precipitación para el norte y centro de México, se muestran resaltadas con relleno de color negro.

Una explicación a dicha respuesta es que la lluvia invernal generalmente es de baja intensidad y al no sobrepasar la capacidad de infiltración queda almacenada en el perfil del suelo para ser utilizada al inicio de la estación de crecimiento (Fritts, 2001). La falta de una asociación significativa entre el crecimiento y la precipitación de verano, se puede atribuir al hecho de que gran parte de la precipitación de verano ocurre cuando el árbol prácticamente ha cesado su división celular. Además, muchas de las lluvias superan la capacidad de infiltración del suelo y escurren como flujo superficial (García, 1978).

Resultados similares se han encontrado para reconstrucciones de precipita- ción en el norte de México y suroeste de EUA (Stahle y Cleaveland, 1993; Stahle et al., 1999; Díaz et al., 2002; Cleaveland et al., 2003; Cerano, 2004 y Villanueva et al., 2007).

\section{Verificación de la reconstrucción}

En los 350 años de precipitación reconstruida, uno de los periodos más secos de la reconstrucción se presentó de 1695 a 1715, lapso en el que ningún año supero la precipitación promedio. En esta sequía se presentaron epidemias intensas de sarampión y viruela que provocaron gran mortalidad entre niños y mujeres de Chihuahua (Dunne, 1958). Información histórica indica que la región 


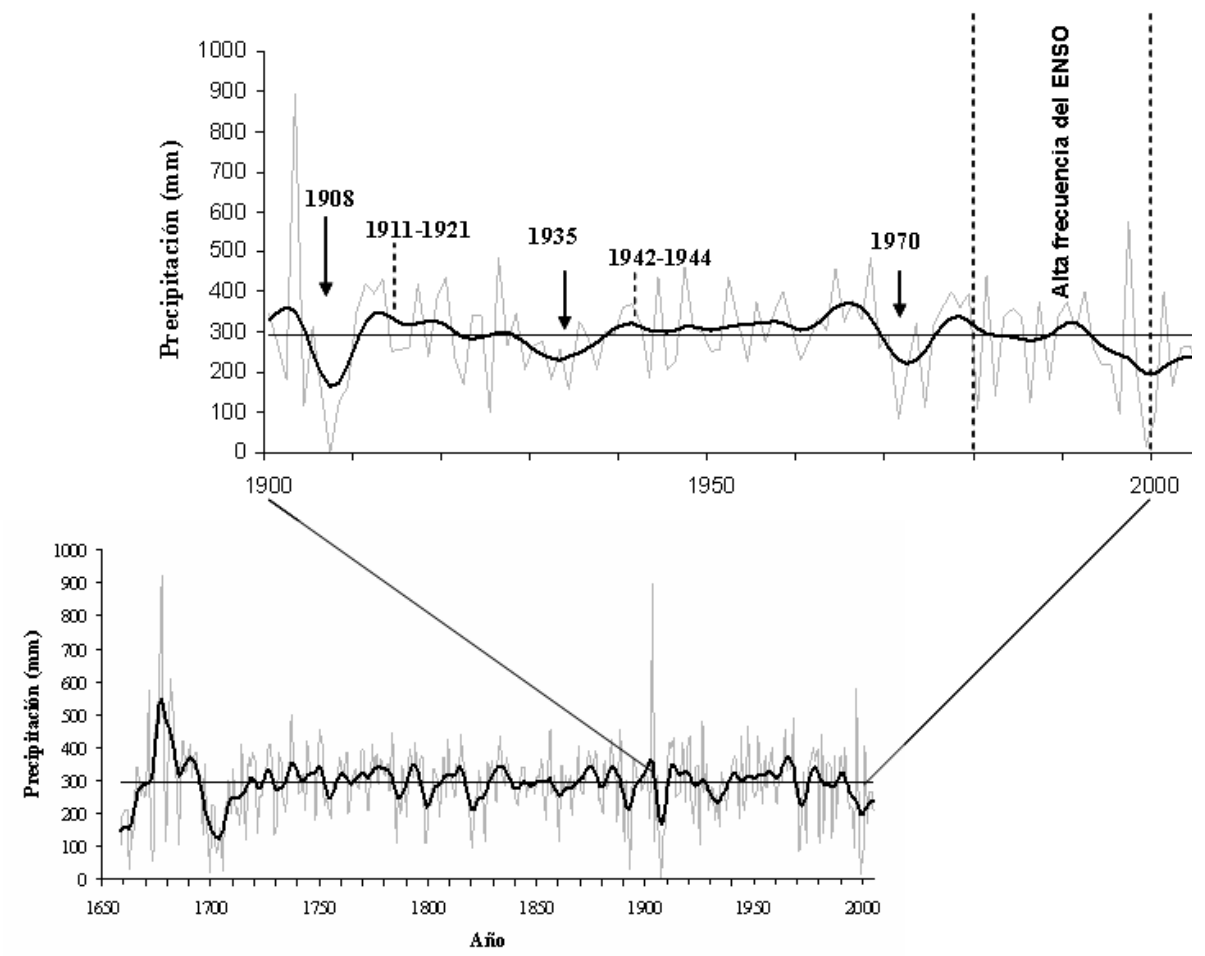

Figura 9. Influencia del ENSO y el MNA en la variabilidad climática del suroeste de Chihuahua, para los siglos XX y XXI. Sequías intensas a consecuencia del efecto de ENSO en su fase calida y períodos con precipitaciones por arriba de la media originadas por efecto del MNA en su fase positiva y ENSO en su fase fría.

oeste del estado sufrió la muerte de 1817 adultos y más de 3000 niños en diversas epidemias acontecidas entre 1690 y 1720 (Gerhard, 1996). Una sequía similar es reportada por Villanueva et al., (2006) para la región del noroeste de Chihuahua y este de Sonora. Esta sequía también estuvo presente en los estados de Durango y Chihuahua (Stahle et al., 1999; Díaz et al., 2002 y Cleaveland et al., 2003).

Los años y periodos secos de 1752 , $1758-1759$ y 1785-1786, provocaron falta de grano, enfermedades y pestes en el estado de Chihuahua (Enfield y Tejedo, 2006). Los años con sequía de 1785 y
1786 conocidos como "El año del hambre", provocaron una hambruna generalizada en el Valle de México, debido a la carestía de alimento (Therrell et al., 2006), afectando estas sequías hasta el norte de México (Florescano y Swam, 1995).

Durante la sequía de 1798-1810 dn el norte y centro de México el descontento social aumentaba debido a los altos precios del maíz; este periodo crítico de sequía es considerado como un importante factor más que contribuyó a la detonación de la Guerra de Independencia (Florescano y Swam, 1995). Este periodo 
también se ha detectado en diversas reconstrucciones paleoclimáticas para el norte, noreste, centro de México y Texas (Stahle y Cleaveland, 1988; Stahle et al., 1999; Therrel et al., 2002; Díaz et al., 2002; Cleaveland et al., 2003; Pohl et al., 2003 y Villanueva et al., 2006).

Otro de los periodos secos en el siglo XIX es el que ocurrió de 1841 a 1870, y que causó severos estragos a la región norte de México. En este periodo ningún año superó la precipitación promedio. Esta sequía se extendió para otras regiones del país como lo constatan reconstrucciones de precipitación estacional de invierno-primavera para Chihuahua y Durango (Stahle et al., 1998; Díaz et al., 2002; Cleaveland et al., 2003), Coahuila (Cerano, 2004) y Nuevo León (Villanueva et al., 2007). Archivos históricos señalan que en los años de la década de 1850, los estados que se vieron más perjudicados debido a las intensas sequías fueron Nuevo León, Coahuila y San Luis Potosí. La situación se complicaba según avanzaba el siglo, para los estados de Coahuila, Chihuahua, Durango, Nuevo León, Sonora y Tamaulipas ante la carestía de alimentos (García, 1997).

La reconstrucción también registra el periodo de 1906 a 1912 con una disminución en precipitación. Los años de 1908 y 1910 se consideran años con sequía severa, en especial 1910 que afectó el norte de México y originó descontento en la población por falta de alimento, lo cual es considerado como un factor más que propició la detonación de la Revolución Mexicana (Florescano, 1980).

A mediados del siglo $X X$ y específicamente en la década de 1950 se presentó un periodo seco que afectó el norte y centro de México. Este periodo seco se ha detectado en reconstrucciones paleoclimáticas para todo el país
(Díaz et al., 2002; Cleaveland et al., 2003; Cerano, 2004 y Villanueva et al., 2006, 2007). Para el caso específico de este estudio la reconstrucción desarrollada no indica la presencia de sequías severas para este periodo; no obstante, el periodo 1924-1941 registró condiciones de precipitación por debajo de la media, constituyendo una intensa sequía con mayor impacto social y económico en esta región que la que se presentó más tarde en la década de 1950 y que fue más generalizada.

Finalmente, el periodo 1971-2005 muestra una sequía prolongada donde solo los años de 1978-1982 y 1992-1993 excedieron la precipitación promedio. La década de 1970 se ha caracterizado como de extrema sequía para el norte de México, en especial los años de 1971, 1972, 1974, 1975, 1976 y 1977 (Florescano, 1980).

Estos eventos secos también se encuentran presentes en reconstrucciones paleoclimáticas para Durango y noreste de México (González, 2003; Cerano, 2004 y Villanueva et al., 2007). La sequía de 1994-2005 representa una de las sequías más intensas de los últimos 300 años para el suroeste del estado de Chihuahua y hasta la fecha continúa afectando la región.

En los tres siglos y medio de precipitación reconstruida se observan fuertes sequías periódicas cada 100 años y sequías menos intensas pero más prolongadas a mitad de siglo. En otras reconstrucciones en el norte de México que se extienden para un periodo más prolongado, se observa un comportamiento similar con sequías intensas a finales de 1500 e inicios de 1600 (Villanueva et al., 2005). La sincronía de las sequías reconstruidas para los últimos 600 años en el norte de México permite inferir que si en el futuro este comportamiento 
continúa, es probable la ocurrencia de intensas sequías en la década del 2050 y finales del 2100; la sequía de finales del siglo $\mathrm{XX}$ aún está presente, aunque para algunas regiones del norte de México como el suroeste de Chihuahua, se ha mostrado con mayor intensidad.

\section{Influencia de patrones circulatorios EI Niño, Oscilación del Sur y el Monzón de Norteamérica}

Las condiciones climáticas de la región de estudio están fuertemente moduladas por fenómenos como el ENSO y el MNA. El MNA produce más del $60 \%$ de la precipitación anual para el suroeste del estado de Chihuahua, en el periodo de verano (ERIC II, 2000). El ENSO propicia un aumento de las lluvias durante el invierno en su fase cálida y una disminución de la precipitación durante el verano, en su fase fría (Niña) ocasiona lluvias normales o por arriba de la media en el verano (Magaña et al., 2003).

Algunos estudios recientes indican que el MNA en la región de Sonora y Sinaloa es débil durante veranos Niño. El inicio del MNA en el noroeste mexicano puede presentar adelantos o retrasos sustanciales (30 días), con respecto a la fecha de inicio de la temporada de lluvias (17 de junio). Un inicio tardío del MNA generalmente significa una temporada de lluvias deficientes, lo cual parece ocurrir en años Niño (Higgins et al., 1999).

\section{Englehart y Douglas (2002)} indican que la región oeste de la Sierra Madre Occidental presenta correlaciones bajas con ENSO, durante la fase positiva de la Oscilación Decadal del Pacifico (PDO, por sus siglas en inglés), fenómeno que es un estimativo de la variabilidad del MNA. En contraste, se presentan correlaciones significativas entre el ENSO y la precipitación durante la fase negativa del PDO.

Eventos intensos de ENSO provocan que la señal del MNA sea negativa, originando una caída en la precipitación y por ende sequías de gran magnitud en el periodo primavera-verano. En contraparte una señal negativa del ENSO propicia condiciones de lluvia normales en el verano, esto atribuido a que no se inhibe o se retrasa la señal del MNA.

La relación de ambos fenómenos es clara en la variabilidad de la precipitación reconstruida para el siglo XX y XXI. La sequía reconstruida en 1908 da inicio debido a los eventos intensos del Niño de 1902, 1904 y 1905; posteriormente se reconstruye un periodo libre de sequías de 1911-1921, atribuible a ningún registro de ocurrencia del fenómeno ENSO, presentándose el MNA en fase positiva. Otra fuerte sequía es la ocurrida en el periodo de 1935 y que fue propiciada por eventos El Niño de 1929, 1930 y 1932; posterior a estos eventos comienza un incremento en la precipitación alcanzando sus puntos máximos en los años de 1942 y 1944, que precisamente son años del Niño en su fase fría, originando un incremento en la precipitación de verano (Figura 9).

La sequía reconstruida para la década de los setenta da inicio a consecuencia del evento de El Niño de 1969, que ocasionó intensas sequías en el verano de 1970. En el invierno de 1970 se registra el mismo fenómeno, que aunque se presentó en su fase fría, no se registran precipitaciones por arriba de la media, dando con esto paso al año más seco de la década de los setentas, el verano de 1971. Finalmente, la constante aparición de El Niño en la década de 1980 y 1990 ha llevado a declarar zonas de desastre a gran parte de los estados del norte del país por la falta de lluvias. 
Los eventos intensos EI Niño 1982-1983, 1986-1987, 1994-1995 y 1997-1998 han ocasionado las intensas sequías reconstruidas para las últimas dos décadas del siglo $X X$ y los años actuales de la primera década del siglo XXI (Figura 9).

\section{CONCLUSIONES}

La similitud o sincronización de eventos secos reconstruidos para Cerro Mohinora y otros sitios del norte y centro de México, permiten determinar la cobertura de los fenómenos climáticos. Las fuertes sequías que afectaron a esta región no son eventos locales, sino que afectan amplias regiones a consecuencia del impacto de patrones de circulación global, lo cual determina la variabilidad de la precipitación en grandes áreas de México.

En los 350 años de precipitación reconstruida se detectan fuertes sequías, tanto al final como al principio de cada siglo, es decir, 1698 a 1718, 1798 a 1807, 1890 a 1908 y 1994 a 2005, así como sequías de menor intensidad pero de mayor duración cada mediados de siglo, como en 1650, 1750, 1850 y la más reciente de 1950. Actualmente, para el suroeste de Chihuahua se vive una sequía que inició en la década de 1990 y continúa hasta el presente. Si esta tendencia continúa se esperaría una sequía en el 2050. No obstante, de 1900 a la fecha las sequías se han repetido cada 20 años; situación que se ha intensificado después de 1970 con sequías cada década, probablemente afectada por una mayor variabilidad de ENSO.

Es fundamental explicar los eventos de baja frecuencia que se han presentado de manera periódica cada 50 o 100 años en el norte de México, y contar con los elementos técnicos para modelar posibles escenarios futuros del clima, lo que es de gran importancia para la planeación del adecuado aprovechamiento de los recursos hídricos con que cuenta esta región.

Los fenómenos circulatorios ENSO y MNA modulan la variabilidad climática de la región del suroeste de Chihuahua. Sin embargo, es importante poner especial énfasis en eventos ENSO, los que actualmente tienen cierta predictibilidad. EI conocimiento con antelación de la incidencia de este fenómeno permitirá inferir la variabilidad hidroclimática para el suroeste del estado de Chihuahua, y tomar las medidas preventivas para tal efecto, particularmente en términos de planeación de los recursos hídricos.

\section{RECONOCIMIENTO}

Esta investigación fue posible gracias al financiamiento otorgado por el Instituto Interamericano para la Investigación del Cambio Climático (IAI), a través del proyecto CRN \# 2047 Documentación, Entendimiento y Proyección de los Cambios en el Ciclo Hidrológico en la Cordillera Americana, a su vez financiado por el US/Nacional Science Foundation (Grant GEO-0452325).

\section{REFERENCIAS}

Bradley, R.S.; H.F. Díaz; J.K. Eischeid; P.D. Jones y P.M. Goodess. 1987. Precipitation fluctuations over Northern Hemisphere land areas since the mid-19th century. Science, 237: 171-175.

Bradley, R.S. 1999. Paleoclimatology. Reconstruction climates of the Quaternary. 2a ed., Academic Press. EUA. 613 pp.

Canadian Environmental Agency. 1997. Environmental Issues. http://www.eei.org/. 
Cerano, P.J. 2004. Reconstrucción de 350 años de precipitación inviernoprimavera para Saltillo, Coahuila. Tesis Profesional. Departamento Forestal. Universidad Autónoma Agraria Antonio Narro, Saltillo, Coahuila, Méx. 152 pp.

Cleaveland, M.K., D.W. Stahle; M.D. Therrell, J. Villanueva-Díaz y B.T. Burns. 2003. Tree-ring reconstructed winter precipitation in Durango, Mexico. Climatic Change 59: 369388.

Cook, E.R. y K. Peters. 1981. The smoothing spline: a new approach to standardizing forest interior tree-ring width series for dendroclimatic studies: Tree-Ring Bulletin 41: 45- 53.

Cook, E.R. 1987. The decomposition of tree-ring series for environmental studies. Tree-Ring Bulletin 47:37-59.

Díaz, C.S., M.D. Therrell, D.W. Stahle y M.K. Cleaveland. 2002. Chihuahua winter-spring precipitation reconstructed from tree-rings, 1647-1992. Climate Research 22: 237-244.

Díaz, H.F., R.S. Bradley y J.K. Eischeid. 1989. Precipitation fluctuations over global land areas since the late 1800's. Journal of Geophysical, Research 94: 1195-1210.

Dunne, P.M. 1958. Las antiguas misiones de los tarahumaras. Editorial Jus, Ciudad de México, 2 vols.

Endfield, G.H. y H.I. Tejedo. 2006. Decades of drought, years of hunger: archival investigations of multiple year droughts in late colonial Chihuahua. Climatic Change 75: 391-419.
Englehart, P. J. y A.V. Douglas. 2002. Mexico's summer rainfall patterns: An analysis of regional modes and changes in their teleconnectivity, Atmosfera, 15: 147-164.

ERIC II. 2000. Instituto Mexicano de Tecnología del Agua. Extractor Rápido de Información Climatológica.

Florescano, E.M. 1980. Análisis histórico de las sequías en México. Secretaría de Agricultura y Recursos Hidráulicos, México.

Florescano, E.M y S. Swan. 1995. Breve historia de la sequía en México. Universidad Veracruzana. México. $246 \mathrm{pp}$.

Fritts, H.C. 1976. Tree-rings and climate. Academic Press, London - New York - San Francisco.

Fritts, H.C. 1991. Reconstructing largescale climatic patterns from tree-ring data. University of Arizona Press, Tucson. 286 pp.

Fritts, H.C. 1999. An Empirical model of the tree-ring response to monthly variations in climate. Laboratory of Tree-Ring Research University of Arizona, Tucson, Arizona, EUA.

Fritts, H.C. 2001. Tree rings and climate. Blackburn Press, Caldwell, New Jersey.

Fujigaki, L.A. y G.A. González. 1982. Epidemias conocidas en México en el siglo XX: Florescano y Malvido (Comps.). Ensayos sobre la historia de las epidemias en México. Instituto Mexicano del Seguro Social. pp. 689-723. 
García, A.V. 1997. Las "sequías" y sus impactos en las sociedades del México decimonónico, 1856-1900. CIESAS, México. 32 pp.

García, E. 1978. Apuntes de climatología. UNAM, México. 153 pp.

Gerhard, P. 1996. La frontera norte de la Nueva España: Universidad Nacional Autónoma de México, Ciudad de México.

Grissino-Mayer, H.D. y T.W. Swetnam. 2000. Century-scale climate forcing of fire regimes in the American Southwest. Holocene 10: 213-220.

Higgins, R.W., Y. Chen y A.V. Douglas, 1999. Interannual variability of the North American warm season precipitation regime. J. Climate 12: 653-680.

Holmes, R.L. 1983. Computer-assisted quality control in tree-ring dating and mesurement. Tree-Ring Bulletin 43: 69-78.

Houghton, J.T., G.K. Jenkins y J.J. Ephramus. 1990. Climate change. The IPCC Scientific Assessment. Cambridge University Press, Cambridge. 364 pp.

IPCC. 1996. Climate change 1995: The Science of Climate Change. Contribution of Working Group I to the Second Assessment Report of the Intergovernmental Panel on Climate Change. J.T. Houghton, L.G. Meira Filho, B. A. Callander, N. Harris, A. Kattenberg and $\mathrm{K}$ Maskell (eds.). Cambridge University Press, Cambridge. 572 pp.

Magaña, V., J.L. Pérez, J.L. Vázquez, E. Carrizosa y J. Pérez. 1999. El Niño y el clima, en: V. Magaña (ed.). Los impactos de El Niño en México. SEP . CONACYT. Mexico. pp. 23-68.

Magaña, V.O., J.L. Vázquez, J.L. Pérez y J.B. Pérez. 2003. Impact of El Niño on precipitation in Mexico. Geofísica internacional 42: 313-330.

Pohl, K., M.D. Therrell, J.S. Blay, N. Ayotte, J.J. Cabrera, S.C. Díaz, E.O. Cornejo, J.A. Elvir; M.E. González, D. Opland, J. Park; G. Pederson, S.S. Bernal, L.S. Vázquez, J. Villanueva-Díaz y D.W. Stahle. 2003. A cool season precipitation reconstruction for Saltillo, Mexico. Tree-ring research Vol. 59(1): 11-19.

Ropelewski, C.F. y M.S. Halpert. 1989. North American precipitation and temperature patterns associated with El Niño/Southern Oscillation (ENSO). Monthly Weather Review 114: 2352-2362.

Stahle, D.W. y M.K. Cleaveland. 1993. Southern oscillation extremes reconstructed from tree-rings of the Sierra Madre Occidental and Southern Great Plains. Journal of Climate 6: 129-140.

Stahle, D.W., D’Arrigo, P.J. Krusic, M.K. Cleaveland, E.R. Cook, R.J. Allan, J.E. Cole, R.B. Dunbar, M.D. Therrell, D.A. Gay, M.D. Moore, M.A. Stokes, B.T. Burns, J. VillanuevaDíaz y L.G. Thompson. 1998. Experimental dendroclimatic reconstruction of the Southern Oscillation. Bulletin of the American Meteorological Society 79(10): 2137-2152.

Stahle, D.W., M.K. Cleaveland, M.D. Therrell y J. Villanueva-Díaz. 1999. Tree-ring reconstruction of winter and summer precipitation in Durango, Mexico, for the past 600 years. 10th Conference of Global Change Studies. Preprint volume, 
American Meteorological Society, 79th Annual Meeting. January 10-15. Dallas, Texas.

Stokes, M.A. y T.L. Smiley. 1968. An Introduction to tree-ring dating. University of Chicago Press, Chicago.

Swetnam, T.W. y C.H. Baisan. 2003. Treering reconstructions of fire and climate history in the Sierra Nevada and southwestern United States. In: T.T. Veblen, W.L. Baker, G. Montenegro, and T.W. Swetnam. Fire and climatic change in temperate ecosystems of the western Americas. Springer-Verlag, Nueva York. pp. 158-195.

Therrell, M.D., D. W. Stahle, J. VillanuevaDíaz, E. Cornejo-Oviedo y M.K. Cleaveland. 2006. Tree-ring reconstructed maize yield in central Mexico: 1474-2001. Climatic Change 74: 493-504.
Villanueva-Díaz, J., J. Cerano-Paredes, D.W. Stahle, M.D. Therrell, L. Vázquez Selem, R. Morán Martínez y B.H. Luckman. 2006. Árboles viejos del centro-norte de México: Importancia ecológica y paleoclimática. Folleto Científico No. 20. INIFAP CENID-RASPA. Gómez Palacio, Durango. 46 pp.

Villanueva-Díaz, J., D.W. Stahle, B.H. Luckman, J. Cerano-Paredes, M.D. Therrell y M.K. Cleaveland. 2007. Winter-spring precipitation reconstructions from tree rings for northeast Mexico. Climate Change 83: 117-131. 